\title{
Compaction of microporous amorphous solid water by ion irradiation
}

\author{
U. Raut, B. D. Teolis, M. J. Loeffler, R. A. Vidal, M. Famá, and R. A. Baragiola \\ Laboratory of Atomic and Surface Physics (LASP), University of Virginia, Charlottesville, Virginia 22904
}

(Received 5 April 2007; accepted 11 May 2007; published online 28 June 2007)

\begin{abstract}
We have studied the compaction of vapor-deposited amorphous solid water by energetic ions at $40 \mathrm{~K}$. The porosity was characterized by ultraviolet-visible spectroscopy, infrared spectroscopy, and methane adsorption/desorption. These three techniques provide different and complementary views of the structural changes in ice resulting from irradiation. We find that the decrease in internal surface area of the pores, signaled by infrared absorption by dangling bonds, precedes the decrease in the pore volume during irradiation. Our results imply that impacts from cosmic rays can cause compaction in the icy mantles of the interstellar grains, which can explain the absence of dangling bond features in the infrared spectrum of molecular clouds. (C) 2007 American Institute of Physics. [DOI: 10.1063/1.2746858]
\end{abstract}

\section{INTRODUCTION}

Ices in astrophysical environments, such as that in the mantles of interstellar grains, are likely accreted from ambient vapor, ${ }^{1}$ a process which, in the laboratory, leads to a microporous amorphous phase at low temperatures $(<100 \mathrm{~K}))^{2,3}$ The microporous structure has a high internal surface area (several hundred $\mathrm{m}^{2} / \mathrm{g}$ ) deduced from the high gas adsorption capacity, which also indicates that most of the pores are accessible to gas molecules from the external environment. $^{3-5}$ The micropores have an infrared absorption signature, weak narrow bands near $2.7 \mu \mathrm{m}$ due to $\mathrm{O}-\mathrm{H}$ stretch vibration of dangling bonds of incompletely coordinated molecules on the pore surfaces. ${ }^{6}$

The microporosity of ice could play an important role in the chemical evolution of interstellar molecular clouds by enhancing the accretion of gas-phase species that can later react and form new molecules. However, the dangling bond absorptions have not been observed to date in the infrared spectra of interstellar water ice, ${ }^{7}$ bringing into question its porosity. A possible reason is processing by cosmic rays since, as shown by Fama et al..$^{8}$ and by Palumbo, ${ }^{9}$ ion irradiation reduces the porosity of microporous ices. To investigate the nature of the compaction, we characterized with three methods the porosity of thin ice films before and after irradiation with $100 \mathrm{keV} \mathrm{Ar}^{+}$ions: (1) optical interference to measure the pore volume, (2) infrared spectroscopy to measure the dangling bond (DB) absorption bands, which are a measure of total pore surface area, and (3) adsorption/ desorption of $\mathrm{CH}_{4}$ gas. The results cast new light on fundamental aspects of irradiation-induced compaction of microporous ice and provide input data needed to model compaction of interstellar ice.

\section{EXPERIMENTAL PROCEDURES}

The experiments were performed in an ultrahigh-vacuum chamber (base pressure $\sim 10^{-10}$ Torr) connected to a massanalyzed ion accelerator. Thin ice films were deposited at $30 \mathrm{~K}$ at the rate of $\sim 10^{15}$ molecules $\mathrm{cm}^{-2} \mathrm{~s}^{-1}$ from high purity water vapor incident at $45^{\circ}$ from an effusive doser onto the gold-mirror surface of a quartz crystal microbalance. The microbalance measures the areal mass of the films, ${ }^{10}$ which is converted to column density in molecules $/ \mathrm{cm}^{2}$ by dividing by the molecular mass. Ices were irradiated at $40 \mathrm{~K}$ with $100 \mathrm{keV} \mathrm{Ar}{ }^{+}$ions at a flux of $\sim 5 \times 10^{11}$ ions $\mathrm{cm}^{-2} \mathrm{~s}^{-1}$, which produces negligible thermal effects on the films. The column density was chosen to be slightly smaller than the maximum ion penetration range, calculated with TRIM. ${ }^{11}$ This was done to avoid alteration of the substrate mirror and contamination of the film by sputtered $\mathrm{Au}$ atoms but leads to a nonuniform energy deposition (and nonuniform compaction) across the film thickness.

Reflectance infrared spectra were collected at $2 \mathrm{~cm}^{-1}$ resolution using a Thermo-Nicolet Nexus 670 spectrometer at $35^{\circ}$ incidence. The spectra were divided by the spectrum of the bare gold mirror to obtain the reflectance $R$ which was then converted to optical depth, $-\ln (R)$. The band areas of dangling bond absorption were obtained after subtracting a base line fit to the continuum absorption.

Reflectance spectra measured at wavelengths between 250 and $600 \mathrm{~nm}$ by an Ocean Optics charge-coupled device spectrometer showed intensity minima and maxima at different wavelengths due to interference between light reflected from the ice-vacuum and the ice-gold interfaces. We fit the reflectance to Fresnel equations ${ }^{12}$ to obtain the index of refraction $n$ and thickness $d$ of the film which, together with the areal mass from the microbalance, gives the average film density $\rho$. We then derive the average film porosity, $1-\rho / \rho_{c}$, using $\rho_{c}=0.94 \mathrm{~g} / \mathrm{cm}^{3}$ for compact ice. ${ }^{13}$

Characterization by gas adsorption was done by exposing the ice films to methane gas and measuring the mass uptake with the microbalance at 40 and $20 \mathrm{~K}$. At $40 \mathrm{~K}$, gas uptake is determined by the competition of adsorption and desorption and therefore we performed gas exposure by increasing the background methane pressure in the chamber in steps, waiting at each step until the mass uptake reached an equilibrium value. At $20 \mathrm{~K}$, desorption is negligible and all the incoming methane is condensed. During this nonequilibrium methane adsorption, we measured changes in the total 


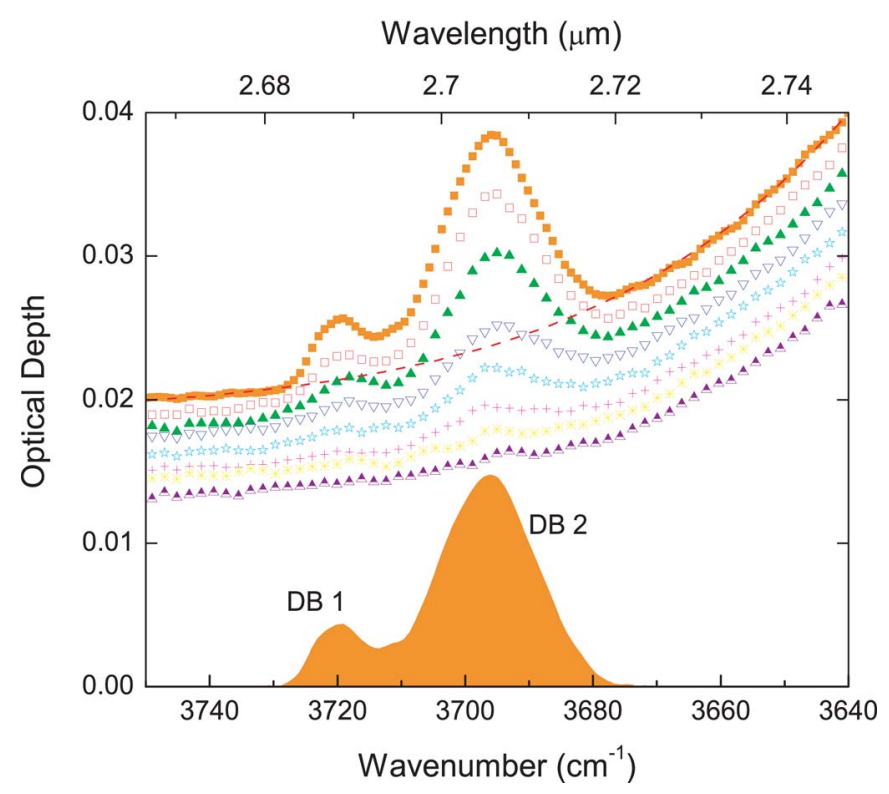

FIG. 1. (Color online) Decrease in the strength of the dangling O-H features in an ice film (initially $\sim 440 \mathrm{~nm}$ thick) during irradiation with $100 \mathrm{keV} \mathrm{Ar}^{+}$ ions at $40 \mathrm{~K}$. The irradiation fluences from top to bottom are $0,0.06,0.18$, $0.62,1.08,2.16,5.19$, and 8.22 in units of $10^{13} \mathrm{ions} / \mathrm{cm}^{2}$. The shaded region shows the dangling bond features in the virgin film after subtracting the continuum base line (dashed red curve).

film thickness by UV-visible interferometry. We point out that that index of refraction of solid methane $e^{14,15}$ in the $250-600 \mathrm{~nm}$ wavelength region is within $0.5 \%$ of that of bulk water. ${ }^{16}$ This means that one could treat the entire film as a single layer during optical analysis, even in cases where methane condenses as an overlayer on top of the ice. At the end of each adsorption experiment performed at $20 \mathrm{~K}$, we warmed the films at $\sim 0.6 \mathrm{~K} / \mathrm{min}$ while measuring the mass loss due to desorption with the microbalance.

\section{RESULTS AND DISCUSSION}

\section{A. Irradiation induced compaction}

Figure 1 shows the decrease in the areas of the dangling O-H absorption bands when irradiating a $440( \pm 10) \mathrm{nm}$ thick $\left(1 \times 10^{18} \mathrm{H}_{2} \mathrm{O} / \mathrm{cm}^{2}\right)$ ice film with $100 \mathrm{keV} \mathrm{Ar}^{+}$ions. The bands at 3720 (DB1) and $3696 \mathrm{~cm}^{-1}$ (DB2) correspond to the $\mathrm{O}-\mathrm{H}$ stretch absorption in doubly and triply coordinated water molecules ${ }^{17}$ on the walls of the pores. The integrated absorbance of these bands is indicative of the total pore surface area. As shown in Fig. 1, both dangling bond bands disappear at the same rate during ion irradiation.

Figure 2 shows the change in porosity and total band area of the dangling bond absorption due to irradiation. It is seen that irradiation compacts the ice from $\rho=0.69$ to $0.92 \mathrm{~g} / \mathrm{cm}^{3}$. We posit that the transient local heating around the path of the ions and the collision cascade started by recoiling water molecules activate the decrease of the internal surface energy of porous ice, as seen in the case of thermally processed ices. ${ }^{18}$ The difference in the fluence dependence of the porosity and of the dangling bond area seen in Fig. 2 shows that irradiation reduces the total surface area faster than the pore volume. This might occur by (i) a process of Ostwald ripening ${ }^{19}$ whereby the coalescence of small pores

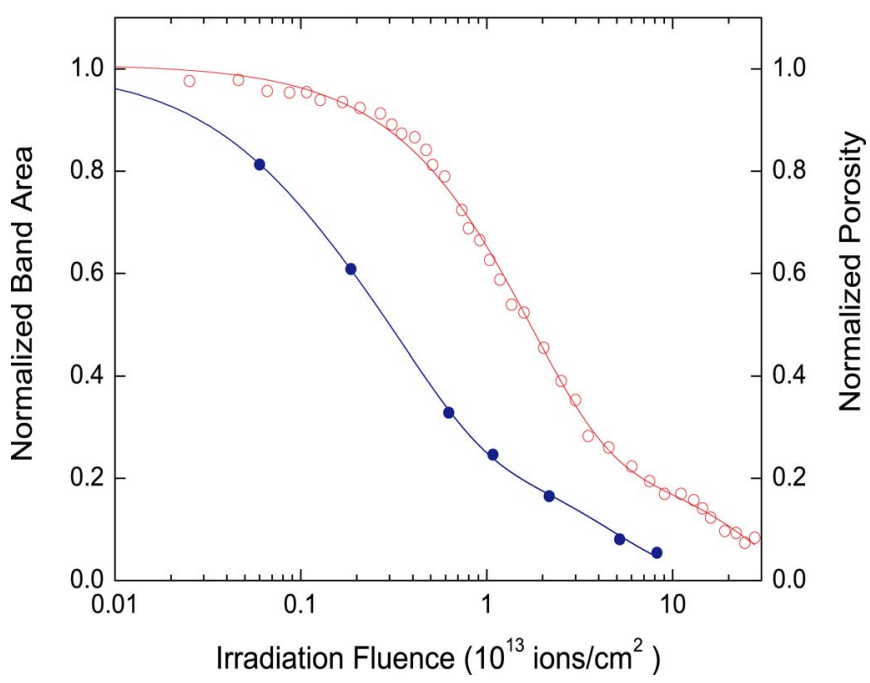

FIG. 2. (Color online) Fluence dependence of ice compaction at $40 \mathrm{~K}$ measured through the reduction of the normalized porosity $(\bigcirc)$ and the normalized total area of the dangling bond adsorptions $(\mathbf{O})$. The lines are meant to guide the eyes.

produces larger ones [Fig. 3(a)], (ii) efficient destruction of smaller pores relative to the larger pores [Fig. 3(b)], or (iii) smoothing of the roughness of the pore walls [Fig. 3(c)]. We note that all three processes lead to an increase in the average pore size and a decrease in the surface energy of the ice. It is also important to note that the absence of the dangling bond infrared absorptions does not necessarily imply the absence of porosity. In the film irradiated to $8.2 \times 10^{13}$ ions $/ \mathrm{cm}^{2}$, the porosity is reduced by $80 \%$, while the dangling bond band area is reduced by $96 \%$. To further elucidate the structural modifications induced by ion irradiation, we now examine the results of our gas adsorption experiments.

\section{B. Gas adsorption}

Figure 4(a) shows the methane uptake at $40 \mathrm{~K}$ versus normalized pressure $P / P_{\max }$, where $P_{\max }=2 \times 10^{-5}$ Torr is the pressure above which continuous condensation of methane occurs on top of the ice $\left(P_{\max }\right.$ is not the saturation vapor pressure $P_{0}$ in usual adsorption isotherms because the gas and solid phases are at different temperatures). The shape of the methane uptake curve in Fig. 4(a) is similar to the type-I gas adsorption isotherm typical of microporous solids, i.e., it rises sharply at low $P / P_{\max }$ to a nearly horizontal plateau. ${ }^{20}$ Ion irradiation decreases the total $\mathrm{CH}_{4}$ uptake but not in proportion to the decrease in porosity and dangling bond absorption area. For instance, a fluence of 3 $\times 10^{12}$ ions $/ \mathrm{cm}^{2}$ reduces the maximum adsorption by $25 \%$ [Fig. 4(a)] but the pore volume by only $13 \%$ (Fig. 2). The large slope of the uptake curve at low pressures signals the presence of high binding energy sites, i.e., proper of pores with dimensions on the order of a few molecular diameters, as expected for vapor-deposited amorphous ice. The high binding energy of the adsorbate and therefore its retention at low pressure is due to the close proximity of the pore walls. ${ }^{20}$ The initial rise in methane uptake is more gradual after irra- 

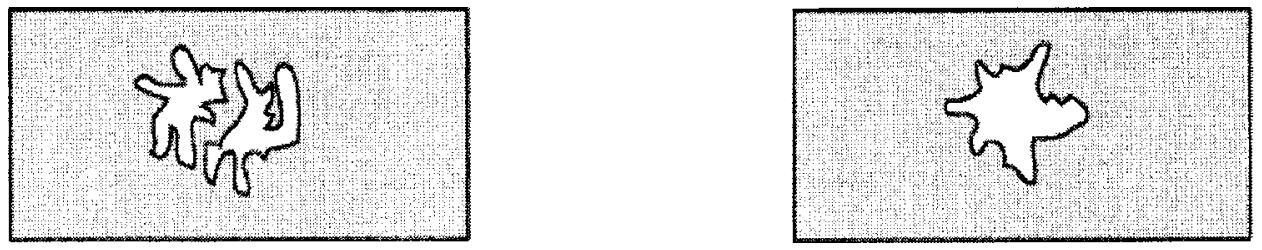

A. Coalesence of micropores
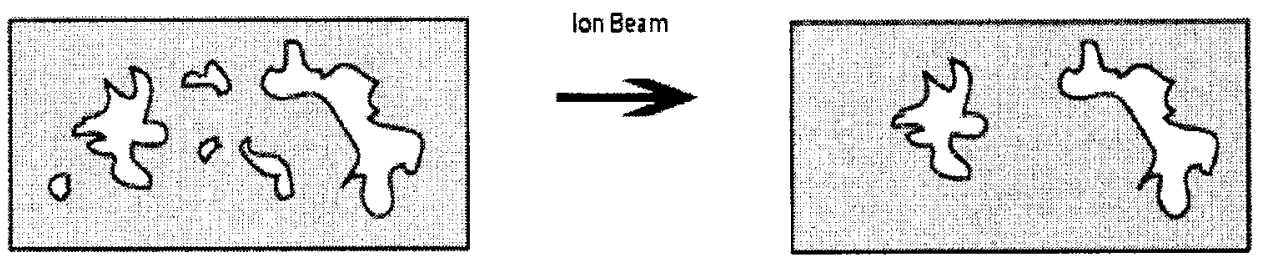

FIG. 3. Sketches showing possible changes in pore structure during compaction. These are cross-sectional slices of the ice film when viewed from the top.

8. Preferential destruction of smaller pores
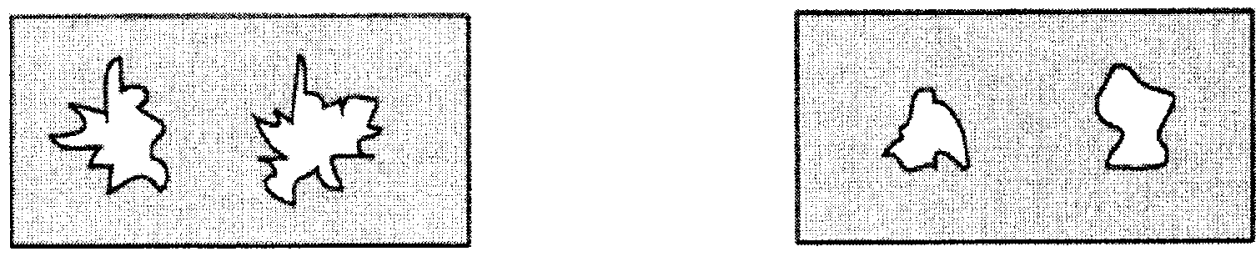

C. Smoothing of pore wall topography

diation, as shown in the normalized plots of Fig. 4(b), indicating a reduction in the number of high binding energy sites such as small pores. ${ }^{20}$

Figure 5 shows the change in total thickness of ice films previously irradiated at $40 \mathrm{~K}$ to different fluences as a function of methane uptake during exposure to a $\mathrm{CH}_{4}$ pressure of

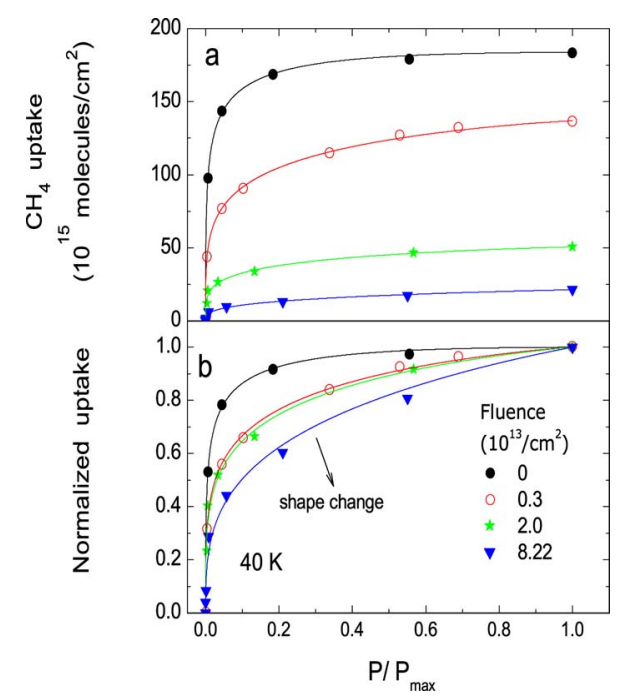

FIG. 4. (Color online) (a) Comparison of $\mathrm{CH}_{4}$ uptake between ice films irradiated at $40 \mathrm{~K}$ to different fluences for different values of $\mathrm{CH}_{4}$ background pressure. $P_{\max }=2 \times 10^{-5}$ Torr is the pressure where continuous multilayer condensation of methane on top of the ice occurs at $40 \mathrm{~K}$. (b) Change in the shape of normalized uptake curves. The lines are meant to guide the eyes.
$5 \times 10^{-8}$ Torr at $20 \mathrm{~K}$, a temperature at which methane can diffuse to the pores but also build multilayers on top of the film. The different films in Fig. 5 have the same column density $\left(1 \times 10^{18} \mathrm{H}_{2} \mathrm{O}\right.$ molecules $\left./ \mathrm{cm}^{2}\right)$, except for a $0.1 \%$ reduction due to sputtering ${ }^{21}$ but their initial thicknesses differ due to compaction by irradiation. The thickness of the unir-

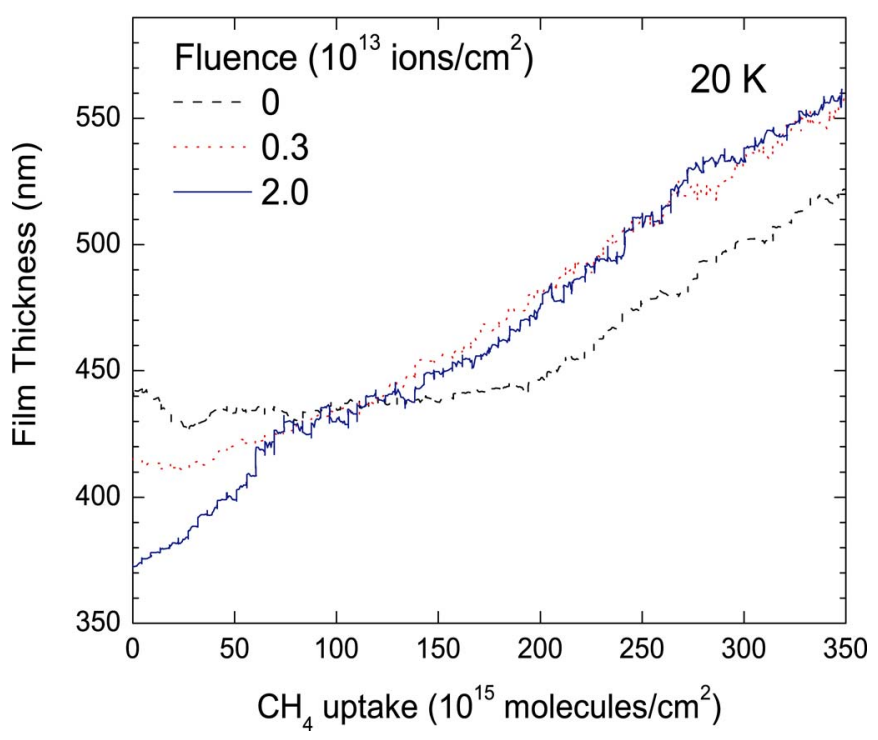

FIG. 5. (Color online) The change in film thickness plotted as a function of methane uptake at $5 \times 10^{-8}$ Torr by ice films at $20 \mathrm{~K}$, which have been ion irradiated at $40 \mathrm{~K}$ to different fluences. The film thicknesses were deduced from UV-visible spectroscopy. The initial film thicknesses are different because irradiation compacts the ice film. 

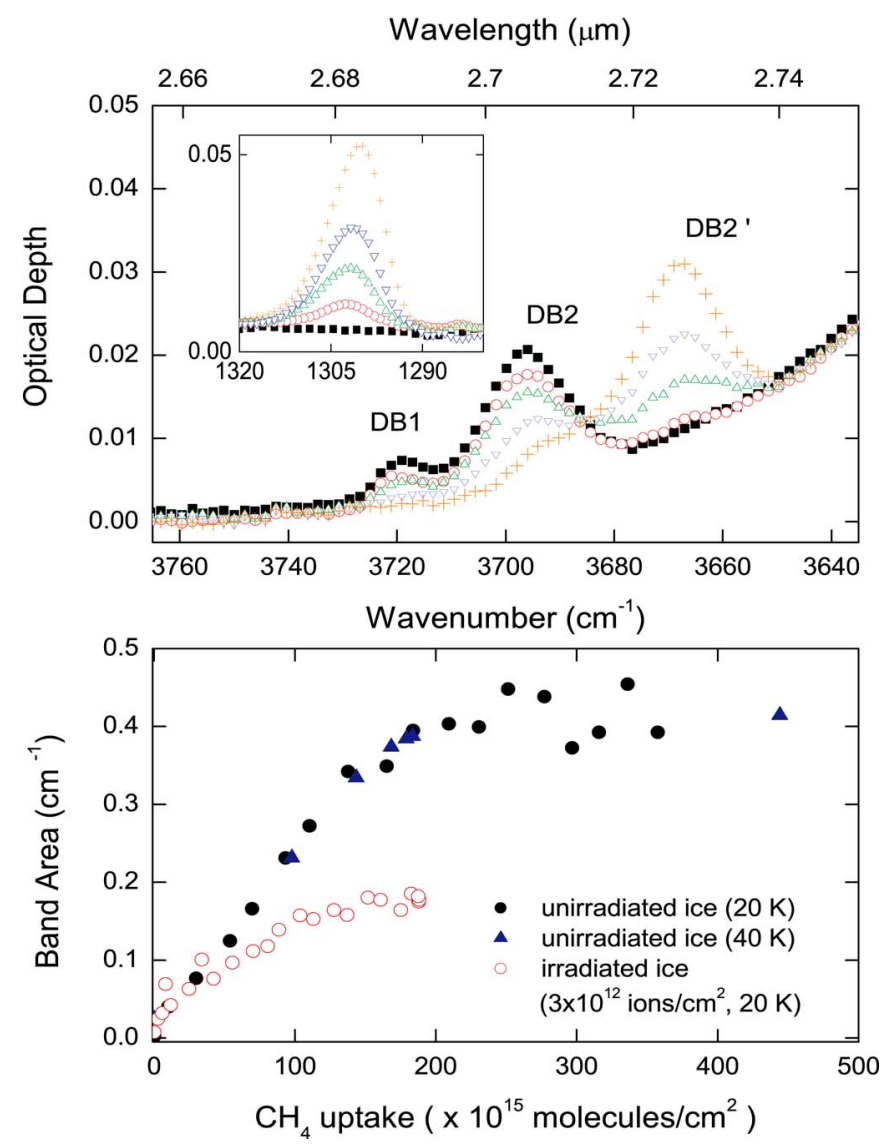

FIG. 6. (Color online) Top: Change in the dangling bonds upon adsorption of methane in unirradiated ice at $20 \mathrm{~K}$. The inset shows the $\sim 1300 \mathrm{~cm}^{-1}$ $\mathrm{CH}_{4}$ band during adsorption. The curves correspond to methane uptake of 0 , 10.0, 53.6, 93.3, and 165.2 in ML units $\left(1 \mathrm{ML}=10^{15} \mathrm{CH}_{4} / \mathrm{cm}^{2}\right)$. Bottom: Increase in the band area of the shifted dangling bond feature at $3669 \mathrm{~cm}^{-1}$ with increasing methane uptake. Gaussian fits were applied to deconvolute the features after subtraction of a curved continuum, as shown in Fig. 1.

radiated film remains constant until the methane uptake exceeds $\sim 200 \times 10^{15} \mathrm{CH}_{4} / \mathrm{cm}^{2}$, an amount sufficient to fill all or most of the pore volume. In contrast, the film irradiated with $3 \times 10^{12} \mathrm{Ar}^{+} / \mathrm{cm}^{2}$ increases in thickness when the uptake exceeds $\sim 40 \times 10^{15} \mathrm{CH}_{4} / \mathrm{cm}^{2}$, while that irradiated to $2 \times 10^{13}$ ions $/ \mathrm{cm}^{2}$ shows an immediate thickness increase. The methane uptake observed before thickness increase, which we call critical uptake, depends on the availability of pores connected to the surface of the film. We note that irradiation with $3 \times 10^{12} \mathrm{Ar}^{+} / \mathrm{cm}^{2}$ lowers the critical uptake by $\sim 80 \%$, indicating a dramatic reduction in pore accessibility which causes methane to accumulate in overlayers on the film surface before or while the pores are filled. The effect is more drastic at an irradiation fluence of $2 \times 10^{13} \mathrm{Ar}^{+} / \mathrm{cm}^{2}$, which lowers the pore accessibility sufficiently to produce an immediate thickness increase upon methane exposure. The inverse of the rate of thickness change with methane uptake gives the density of solid methane, $0.52 \pm 0.01 \mathrm{~g} / \mathrm{cm}^{3}$, the same value as previously reported. ${ }^{14,22}$

Methane adsorption causes a reduction of DB1 and DB2 while at the same time two new dangling bond absorption bands emerge, blueshifted by $30 \mathrm{~cm}^{-1}$ (Fig. 6). This shift in DB position has been seen previously. ${ }^{5}$ The area of the shifted DB2 absorption peak (DB2') at $3669 \mathrm{~cm}^{-1}$ increases

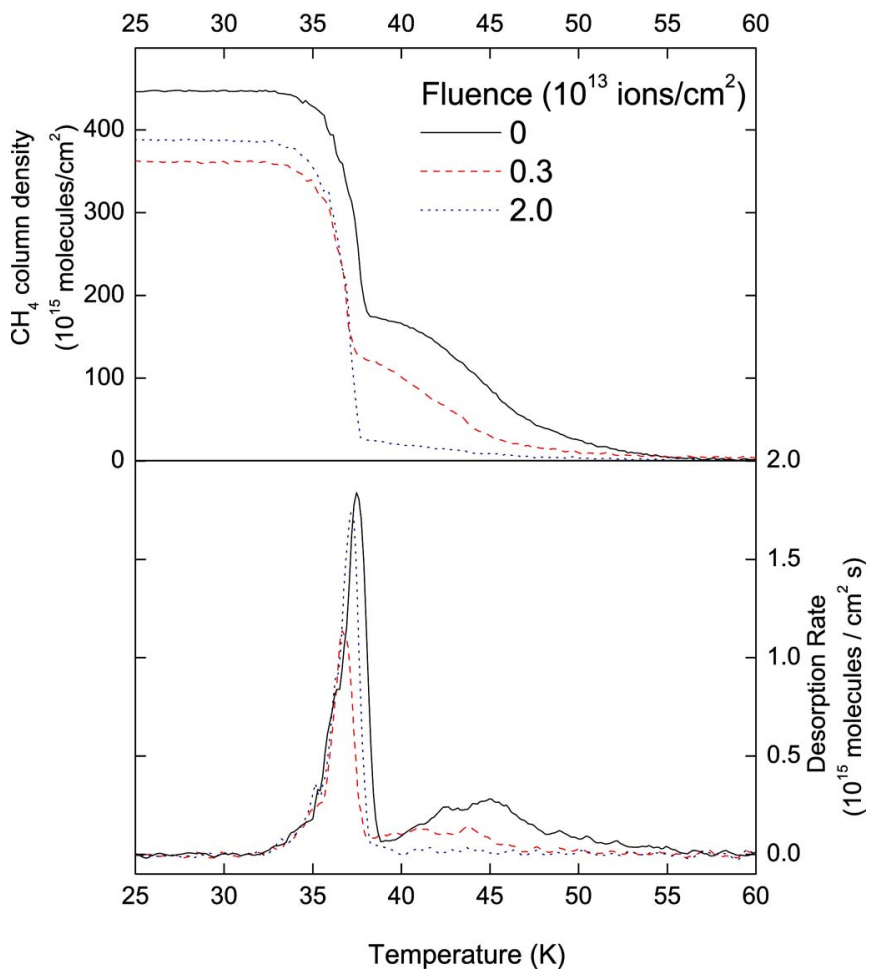

FIG. 7. (Color online) Thermal desorption spectra of methane adsorbed (at $20 \mathrm{~K}$ ) onto unirradiated and irradiated ice films. Films were warmed at $\sim 0.6 \mathrm{~K} / \mathrm{min}$ in vacuum. The first drop in the areal mass beginning at $32 \mathrm{~K}$ is due to the loss of the methane overlayer. The second decrease in the areal mass is due to loss of methane occupying the pore volume of the ice. The desorption rates in bottom panel were obtained by numerical differentiation of the graphs in the top panel.

linearly with methane uptake onto unirradiated ice at 20 or $40 \mathrm{~K}$ and saturates after the uptake exceeds 200 $\times 10^{15} \mathrm{CH}_{4} / \mathrm{cm}^{2}$. The saturation uptake matches the critical uptake for unirradiated ice film at $20 \mathrm{~K}$ (Fig. 5).

\section{Thermal desorption}

Following gas adsorption at $20 \mathrm{~K}$, the films were warmed at $\sim 0.6 \mathrm{~K} / \mathrm{min}$. In Fig. 7 , we show the total change in areal mass and desorption rate during warming. The first desorption peak at $\sim 37 \mathrm{~K}$ is due to desorption of the methane overlayer. This is followed by a broad $(\sim 38-55 \mathrm{~K})$ desorption peak of methane escaping from the internal pores, indicating a distribution of binding energies. We note that the film irradiated to $2 \times 10^{13} \mathrm{Ar}^{+} / \mathrm{cm}^{2}$ also shows a small amount $\left(\sim 27 \times 10^{15} / \mathrm{cm}^{2}\right)$ of methane desorption from the pores in spite of our observation at $20 \mathrm{~K}$ (based on the film thickness measurements of Fig. 5) that all the methane was accumulated as an external overlayer. Thus, for a fluence of $2 \times 10^{13} \mathrm{Ar}^{+} / \mathrm{cm}^{2}$, methane is sufficiently mobilized during warming to reach pores previously inaccessible at $20 \mathrm{~K}$. Above $60 \mathrm{~K}$, we do not detect methane $\left(<5 \times 10^{14} \mathrm{CH}_{4} / \mathrm{cm}^{2}\right)$ irrespective of the irradiation fluence.

\section{Astrophysical implications}

Mayer and Pletzer ${ }^{3}$ have proposed that interstellar water ice is microporous but so far there have been no reports on the unambiguous observation of the infrared dangling bond 
features in interstellar ices that would signal the existence of microporosity. Keane et al. detected a slight feature at $3660.5 \mathrm{~cm}^{-1}$ with a full width at half maximum of $10 \mathrm{~cm}^{-1}$ in the spectrum of interstellar dust, ${ }^{23}$ but the low signal to noise ratio did not allow a conclusive identification. Other evidence presented for or against the presence of the dangling bonds on interstellar ice has been indirect. $\mathrm{CO}$ has been detected in line of sight of different interstellar objects through its absorption bands at 2139 and $2136 \mathrm{~cm}^{-1}$ but without the $2152 \mathrm{~cm}^{-1}$ band due to $\mathrm{CO}$ absorbed on dangling O-H sites. ${ }^{24}$ The absence of this band has led to the hypothesis that the dangling bonds are inaccessible to $\mathrm{CO}$ because of competitive adsorption from other species. ${ }^{24}$ However, our work suggests a more direct explanation, destruction of the dangling bond sites to below detection limits by the impact of cosmic rays or stellar winds.

\section{SUMMARY AND CONCLUSIONS}

In summary, infrared spectroscopy, UV-visible spectroscopical interferometry, and $\mathrm{CH}_{4}$ adsorption were used to understand the structural changes induced in porous ice films by ion irradiation. The main findings are the following.

(i) The surface area of porous ice decreases at a faster rate than the pore volume during ion-induced compaction.

(ii) The absence of dangling bond features does not imply a fully compact ice.

(iii) The change in the shape of the normalized $\mathrm{CH}_{4}$ uptake curve with increasing irradiation fluence indicates the formation of adsorption sites with lower binding energy, likely associated with an increase in pore size.

(iv) The gas adsorption ability of the ice is reduced with irradiation but not in proportion with porosity or the surface area as measured by the dangling bond infrared absorptions.

(v) Ion irradiation reduces pore accessibility for foreign gas molecules as indicated by the dramatic decrease in the critical uptake value with increasing ion fluence at $20 \mathrm{~K}$. This effect is enhanced at lower temperature owing to the decreased mobility of methane.

(vi) For unirradiated films the band area of the dangling bond feature at $3669 \mathrm{~cm}^{-1}$ (DB2') increases linearly with methane uptake and remains constant after the critical uptake at which point $\mathrm{CH}_{4}$ starts condensing as an overlayer increasing the total film thickness.

(vii) Ice compacts at fluences that are low compared to those that cause significant sputtering.
These results imply that impacts from cosmic rays can compact the icy mantles of interstellar grains and thus explain the absence of dangling bond features in the infrared spectra of molecular clouds. The estimation of the time required for compaction will require knowledge of the cosmic ray flux and also further experiments to determine how the compaction process depends on the type and energy of the ion.

\section{ACKNOWLEDGMENTS}

This research was supported by NASA Origins of the Solar System and NSF Astronomy programs.

\footnotetext{
${ }^{1}$ R. Papoular, Mon. Not. R. Astron. Soc. 362, 489 (2005).

${ }^{2}$ R. A. Baragiola, in Water In Confining Geometries, edited by J. P. Devlin and V. Buch (Springer, Berlin, 2003); K. P. Stevenson, G. A. Kimmel, Z. Dohnalek, R. S. Smith, and B. D. Kay, Science 283, 1505 (1999); Z. Dohnalek, G. A. Kimmel, P. Ayotte, R. S. Smith, and B. D. Kay, J. Chem. Phys. 118, 364 (2003); M. S. Westley, G. A. Baratta, and R. A. Baragiola, ibid. 108, 3321 (1998).

${ }^{3}$ E. Mayer and R. Pletzer, Nature (London) 319, 298 (1986).

${ }^{4}$ A. Bar-Nun, J. Dror, E. Kochavi, and D. Laufer, Phys. Rev. B 35, 2427 (1987); P. Ayotte, R. S. Smith, K. P. Stevenson, Z. Dohnalek, G. A. Kimmel, and B. D. Kay, J. Geophys. Res. 106, 33387 (2001).

${ }^{5}$ N. Horimoto, H. S. Kato, and M. Kawai, J. Chem. Phys. 116, 4375 (2002).

${ }^{6}$ J. P. Devlin and V. Buch, J. Phys. Chem. 99, 16534 (1995).

${ }^{7}$ J. V. Keane, A. C. A. Boogert, A. G. G. M. Tielens, P. Ehrenfreund, and W. A. Schutte, Astron. Astrophys. 375, L43 (2001).

${ }^{8}$ M. Fama, D. M. Pugh, B. D. Teolis, and R. A. Baragiola, Proceedings of the DPS, 2001 (unpublished); U. Raut, M. J. Loeffler, R. A. Vidal, and R. A. Baragiola, 35th Lunar and Planetary Science Conference, Abstract \#1922 (2004) (unpublished).

${ }^{9}$ M. E. Palumbo, Astron. Astrophys. 453, 903 (2006).

${ }^{10}$ N. J. Sack and R. A. Baragiola, Phys. Rev. B 48, 9973 (1993).

${ }^{11}$ J. F. Ziegler and J. P. Biersack, SRIM 2003, stopping and range of ions in matter, 2006 (available at www.srim.org).

${ }^{12}$ O. S. Heavens, Optical Properties of Thin Solid Films (Dover, New York, 1991); B. D. Teolis, M. J. Loeffler, U. Raut, M. Famá, and R. A. Baragiola (Icarus, in press).

${ }^{13}$ A. H. Narten, C. G. Venkatesh, and S. A. Rice, J. Chem. Phys. 64, 1106 (1976).

${ }_{14}$ J. V. Martonchik and G. S. Orton, Appl. Opt. 33, 8306 (1994).

${ }^{15}$ G. S. Orton and J. V. Martonchik (private communication).

${ }^{16}$ S. G. Warren, Appl. Opt. 23, 1206 (1984).

${ }^{17}$ V. Buch and J. P. Devlin, J. Chem. Phys. 94, 4091 (1991).

${ }^{18}$ J. A. Ghormley, J. Chem. Phys. 48, 503 (1968).

${ }^{19}$ J. Schmelzer, T. J. Moller, and V. V. Slezov, J. Phys. Chem. Solids 56, 1013 (1995).

${ }^{20}$ F. Rouquerol, J. Rouquerol, and K. Sing, Adsorption by Powders and Porous Solids: Principles, Methodology and Applications (Academic, San Diego, CA, 1999).

${ }^{21}$ R. A. Baragiola, Philos. Trans. R. Soc. London, Ser. A 362, 29 (2004).

${ }^{22}$ G. Mulas, G. A. Baratta, M. E. Palumbo, and G. Strazzulla, Astron. Astrophys. 333, 1025 (1998)

${ }^{23}$ J. V. Keane, A. C. A. Boogert, A. G. G. M. Tielens, P. Ehrenfreund, and W. A. Schutte, Astron. Astrophys. 375, L43 (2001).

${ }^{24}$ H. J. Fraser, M. P. Collings, J. W. Dever, and M. R. S. McCoustra, Mon.
} Not. R. Astron. Soc. 353, 59 (2004). 